\title{
Evanescent wave interference and the total transparency of a warm high-density plasma slab
}

\author{
E. Fourkal ${ }^{1}$, I. Velchev ${ }^{1}$, C-M. Ma ${ }^{1}$, and A. Smolyakov ${ }^{2}$ \\ ${ }^{1}$ Department of Radiation Physics, Fox Chase Cancer Center, \\ Philadelphia, PA 19111, U.S.A. and \\ ${ }^{2}$ Department of Physics and Engineering Physics, \\ University of Saskatchewan, Saskatoon, Canada
}

(Dated: August 29, 2018)

\begin{abstract}
It is shown that an overcritical density plasma slab can be totally transparent to a $p$-polarized obliquely incident electromagnetic wave. High transparency is achieved due to the interference of the evanescent waves in the subcritical region. The transmission coefficient has the resonant character due to the excitation of a plasma surface mode (plasmon-polariton). It is crucial that two evanescent waves have certain phase shift to provide non-zero energy flux through non-transparent region. The required phase shift is obtained at the exact resonance and corresponds to the absolute transparency. In a warm plasma case, the excitation of the propagating longitudinal (electrostatic) modes becomes possible. We demonstrate that these longitudinal excitations facilitate the total transparency of an opaque plasma slab creating additional resonances in the transmission property of the system.
\end{abstract}

PACS numbers: 52.27.-h, 52.25.Os, 52.38.Dx, 52.35.Lv, 52.38.-r 


\section{INTRODUCTION}

There exists a large number of theoretical and experimental studies dedicated to the problem of light interaction with dense media having negative dielectric permittivity [1, 2, 6, 8, 13]. The increasing interest in the properties of such media has been driven by their potential applications in different branches of science and technology. Understanding of the fundamental processes that take place during the interaction of electromagnetic radiation with overcritical density plasmas $\left(\epsilon=1-\omega_{p}^{2} / \omega^{2}<0\right.$ for $\left.\omega<\omega_{p}\right)$ is of great importance to a number of areas. In particular, one of the challenges of the inertial confinement fusion (ICF) experiments is the effective heating of the fuel pellet by the laser light (in the direct drive) or X-rays (in the indirect drive ignition experiments). The two well known absorption mechanisms in plasma are of collisional [3, 16] and resonant [4, 9, 10] nature. In the collisional absorption process, the energy of light is converted into thermal electron motion through electron-ion collisions. In the resonant absorption mechanism, the linear conversion in nonuniform plasma leads to a resonant excitation of electrostatic plasma wave, which subsequently decays giving rise to energetic electrons.

In this work we investigate another resonant mechanism that dramatically enhances the penetration of the electromagnetic field into overdense plasma. This mechanism involves surface plasma wave or plasmon-polariton [12, 19] which may be excited at the plasmaplasma interface. Unlike bulk plasma waves, which are electrostatic in nature, the surface waves are of mixed longitudinal-transverse type and can couple to radiating electromagnetic fields. As was shown in Ref. 1], at certain conditions the energy of the incident electromagnetic radiation can be completely absorbed by the plasma through the resonant excitation of surface plasma waves which subsequently dissipate through standard irreversible processes like linear wave transformation or collisions.

In this paper we show that the resonant excitation of surface modes may create the condition for absolute transparency of an overdense plasma slab. The transparency mechanism studied in our work is directly related to the problem of the anomalous transparency of light in materials with negative permittivities. Media with negative electric permittivity and magnetic permeability [20] (the so-called left-handed materials or LHM) possess some unusual optical properties, in particular, the possibility of creating an "ideal lens" offering sub-wavelength resolution [8, 17, 18]. As was pointed out by Pendry 17], the superlens 
is essentially based on the possibility of evanescent waves amplification, facilitated by the excitation of surface plasmons. The amplification of the evanescent waves and their interference are the basic mechanisms behind the anomalous transparency demonstrated in our work. This indicates the feasibility of superlensing in materials without any restrictions on the magnetic permeability $\mu$, which has also been noted by Pendry.

In Ref. 21], the authors suggested that the incident electromagnetic wave may excite a pair of coupled surface plasma waves on both sides of the slab. The energy of the surface wave running on the opposite side of the slab can be dissipated by reemission of the electromagnetic waves, which causes the layer to appear totally transparent. However a special symmetric structure consisting of media with different dielectric permittivities has to be formed in order for a pair of coupled surface waves to exist.

In this paper we reexamine the conditions for total transparency of a layer of overcritical density plasma. Anomalously high transmission of light is explained through the interference of the evanescent fields formed by the incident/emitted electromagnetic radiation and the field of the surface plasma wave. We show that the total transparency of light can be achieved even when a single surface plasma mode is excited. Therefore, there is no need to form a symmetric structure in which an overcritical density plasma slab is sandwiched between two additional layers with specially chosen dielectric permittivities. In other words anomalous light transmission can be achieved by passing the light through a single plasmaplasma interface. In most laboratory experiments when high-power laser light interacts with a solid density target, a prepulse precedes the main laser pulse, creating the plasma density profiles discussed in this paper.

\section{SURFACE WAVE ASSISTED TOTAL LIGHT TRANSMISSION IN COLD PLASMAS}

As it was noted above, high transparency of dense plasma occurs as a result of the resonant excitation of a surface mode. Since the surface of the medium with $\epsilon<0$ behaves

like surface wave resonator [2], the problem of surface wave excitation is equivalent to that of resonator excitation. Under resonant conditions (external field frequency and its wave vector's tangential component have to match those determined from the dispersion relation for the plasma surface wave) the coupling between the surface eigenmode and the external 
field becomes extremely efficient. The minimum in the reflection coefficient (the maximum in the transmission coefficient in the absence of dissipation) occurs for these resonant values of the external wave frequency and wave number.

Here we review a dispersion relation for surface waves that exist on a cold plasma-plasma interface for arbitrary values of dielectric permittivities of the adjacent regions. It is found by solving the linearized set of cold fluid-Maxwell equations,

$$
\begin{array}{r}
\nabla \times \mathbf{E}=-\frac{1}{c} \frac{\partial \mathbf{B}}{\partial t} \\
\nabla \times \mathbf{B}=-\frac{4 \pi n_{0} e}{c} \tilde{\mathbf{v}}+\frac{1}{c} \frac{\partial \mathbf{E}}{\partial t} \\
\frac{\partial \tilde{\mathbf{v}}}{\partial t}=-\frac{e}{m} \mathbf{E},
\end{array}
$$

where ions are assumed to be motionless. Letting all physical quantities vary as $\boldsymbol{\Psi}(x) \exp \left(i k_{y} y-i \omega t\right)$, where $x$ is the coordinate into the plasma and $y$ runs along the interface with the field components of the form $\mathbf{E}=\left(E_{x}, E_{y}, 0\right), \mathbf{B}=\left(0,0, B_{z}\right)$ one obtains,

$$
\epsilon \frac{d}{d x}\left(\frac{1}{\epsilon} \frac{d B_{z}}{d x}\right)-\alpha^{2} B_{z}=0
$$

where $\alpha^{2}=\left(k_{y}^{2}-\frac{\omega^{2}}{c^{2}} \epsilon\right)$ and $\epsilon=1-\omega_{p}^{2} / \omega^{2}$. Assuming that the interface is located at the plain $x=0$, and for $x<0$ plasma has a constant density such that $\omega_{p}=\omega_{p 1}$, and for $x>0$ a different constant density $\omega_{p}=\omega_{p 2}$, the solution to Eq. (2) in both regions is,

$$
\begin{array}{r}
B_{z}=A_{1} \exp \left(\alpha_{1} x\right), x<0 \\
B_{z}=A_{2} \exp \left(-\alpha_{2} x\right), x>0
\end{array}
$$

It should be noted that here we only keep those solutions that decay away from the interface (a condition for existence of a well-defined surface mode). Using the boundary conditions at $x=0$ requiring the continuity of $B_{z}$ and $d B_{z} / d x / \epsilon$, one readily obtains the dispersion relation for the surface waves on the cold plasma-plasma interface,

$$
\alpha_{1} / \epsilon_{1}+\alpha_{2} / \epsilon_{2}=0 \rightarrow k_{y}=\frac{\sqrt{\left(\Delta-\omega^{2}\right)\left(\omega^{2}-1\right)}}{\sqrt{1+\Delta-2 \omega^{2}}}
$$

where $\Delta=\omega_{p 1}^{2} / \omega_{p 2}^{2}, \omega$ is normalized to the plasma frequency in the region 2 where $\epsilon<0$ and $k_{y}$ is normalized to the classical skin depth $\delta=c / \omega_{p 2}$. As can be seen from Eq. (3), $\epsilon_{1}$ and $\epsilon_{2}$ must have opposite signs, so that one plasma region must have undercritical electron density while the other-overcritical. 
One important difference between the surface plasmons running on a plasma-vacuum interface (obtained from (3) by setting $\Delta=0)$ ) and those on a plasma-plasma interface lies in a fact that the phase velocity in the latter case can exceed that of light, whereas the phase velocity of the former is always subluminal. Because of this fact, the surface plasmons on a plasma-plasma interface can couple to radiating electromagnetic fields, which are evanescent in plasma but may become propagating if a vacuum region is present beyond plasma layers. The opposite is also true: if an external $p$-polarized electromagnetic pulse is obliquely incident on the structure comprising two plasma layers, it may under a resonant condition excite a surface plasma mode. On the contrary, a $p$-polarized electromagnetic pulse obliquely incident on a vacuum-plasma interface cannot excite a plasma surface wave since the phase velocity of this collective mode can never exceed the speed of light (the perturbation of the plasma surface by the external field propagates in this case with phase velocity greater than that of light) and the field of the external radiation is not evanescent (as we mentioned earlier, the fields of the surface wave are decaying away from the interface, or only evanescent external fields can excite the plasma surface mode). This means that if a layer of dielectric is added, so that a system comprising dielectric medium, vacuum and overcritical density plasma is formed in such a way that the external field in vacuum is evanescent (through total internal reflection of light in dielectric material) and the perturbation of the plasma surface by the evanescent field propagates with the phase velocity that is lower than the speed of light, then the external electromagnetic field may excite the surface plasma mode on a vacuum-plasma interface [2, 6].

Optical properties of a double-plasma system are determined by matching the fields of the incident/reflected electromagnetic waves with those of the surface wave. Let us consider a system which is characterized by spatial density (dielectric constant) distribution shown in Fig. (11). A $p$-polarized plane electromagnetic wave is obliquely incident on this structure. In both plasma regions, the electromagnetic field $\mathbf{B}=\left(0,0, B_{z}\right)$ is the solution to the following equation:

$$
\epsilon \frac{d}{d x}\left(\frac{1}{\epsilon} \frac{d B_{z}}{d x}\right)+\frac{\omega^{2}}{c^{2}}\left(\epsilon-\sin ^{2} \theta\right) B_{z}=0,
$$

with a general solution having the following from,

$$
B_{z}=A_{1} e^{i k x}+A_{2} e^{-i k x}
$$

where $k=k_{0} \sqrt{\left(\epsilon-\sin ^{2} \theta\right)}, k_{0}=\omega / c$. The electromagnetic fields in the vacuum regions 
$(x<-d$ and $x>a)$ have the following form,

$$
\begin{array}{r}
B_{z}=E_{0} e^{i k_{0} \cos \theta x}+R e^{-i k_{0} \cos \theta x}, x<-d \\
B_{z}=T e^{i k_{0} \cos \theta x}, \quad x>a
\end{array}
$$

where $T$ and $R$ designate the field components for the transmitted and reflected waves. Matching solutions at different boundaries by requiring continuity of $B_{z}$ and $d B_{z} / d x / \epsilon$ across interfaces, one obtains a system of six equations (not shown here) for the unknown coefficients. The expression for the transmission coefficient has the following form,

$$
\begin{gathered}
T=\left|\frac{4 e^{i\left(k_{1} d+k_{2} a-(a+d) k_{0} \cos \theta\right)} k_{0} k_{1} k_{2} \epsilon_{1} \epsilon_{2} \cos \theta}{\left(\left(1+e^{2 i k_{1} d}\right) k_{0} k_{1} k_{2} \epsilon_{1} \epsilon_{2} \cos \theta+e^{2 i k_{2} a}\left(1+e^{2 i k_{1} d}\right) k_{0} k_{1} k_{2} \epsilon_{1} \epsilon_{2} \cos \theta-2 i e^{i\left(k_{1} d+k_{2} a\right)}\right)}\right|^{2} \\
\Upsilon=k_{2} \epsilon_{2} \cos \left[k_{2} a\right]\left(k_{1}^{2}+k_{0}^{2} \epsilon_{1}^{2} \cos ^{2} \theta\right) \sin \left[k_{1} d\right]+ \\
\left(k_{1} \epsilon_{1} \cos \left[k_{1} d\right]\left(k_{2}^{2}+k_{0}^{2} \epsilon_{2}^{2} \cos ^{2} \theta\right)-i k_{0}\left(k_{2}^{2} \epsilon_{1}^{2}+k_{1}^{2} \epsilon_{2}^{2}\right) \cos \theta \sin \left[k_{1} d\right]\right) \sin \left[k_{2} a\right],
\end{gathered}
$$

where $k_{1,2}=k_{0} \sqrt{\left(\epsilon_{1,2}-\sin ^{2} \theta\right)}$. For resonant values of the external electromagnetic field frequency and incidence angle, one should expect anomalously high transmission of light.

Figure (21) shows the transmission coefficient as a function of the incidence angle. As one can see, there is a sharp increase in the transmission properties of the system when the angle of incidence matches one of many resonant values of $\theta=0.671955$ radians at which point the transmission coefficient reaches the value $T=0.99999$. The resonant values exactly correspond to those given by an expression (3) for the dispersion relation of the surface plasma wave. Thus, the anomalous transmission occurs even for the system consisting of an undercritical density plasma layer adjacent to an overcritical density plasma slab, so that there is no need to form a sandwich-like structure as argued in Refs. ([2, 6, 7]) in which a second surface plasma wave has to be excited on the opposite side of the overcritical density layer to achieve the same effect. In other words, the anomalous light transmission can be achieved through the excitation of a single surface plasma wave. It is interesting to note that for a sandwich-like system, there are generally two peaks in the transmission coefficient at two different but close angles of incidence[21]. When the second layer of undercritical plasma is removed, or the thickness of the overcritical density plasma slab becomes too large, the two solutions degenerate into one, which corresponds to the surface plasmon running on a single plasma-plasma interface. Analogous effect is seen in quantum mechanics where the 
energy level of a particle in two-humped potential is split into two energy states that are close to each other 15]. As the potential is transformed into a single well, the two energy levels degenerate into a single energy state.

As one can see from Figures (3) and (4) the light transmission is a non-monotonous function of the widths of both plasma layers with a single maximum reached at certain correlated values. These values are determined by the interference condition between the two non-propagating (evanescent) fields. The evanescent fields are expressed through the hyperbolic functions (anomalous transmission occurs when both $k_{1}$ and $k_{2}$ take on pure imaginary values). It can be easily seen that the total field in both plasma regions constitutes a sum of two exponential functions, one decaying with distance $e^{-x}$ and the other growing $e^{x}$ (x points in the direction from left to right). It is a known fact that a single evanescent (decaying) wave carries no power. It can be easily shown that the $x$ component of the Poynting vector for this wave is $S_{x} \sim R e[k]=0$ ( $k$ is a purely imaginary spatial decay constant). However, when the total field is a superposition of decaying and growing modes, $E, B \sim A_{1} \exp [-i k x]+A_{2} \exp [i k x]$ ( $k$ is purely imaginary), the $x$ component of the Poynting vector $S_{x}$ becomes,

$$
S_{x}=\frac{1}{2} \operatorname{Re}\left[E_{y} B_{z}^{*}\right] \sim \operatorname{Re}\left[k\left(A_{1} A_{2}^{*}-A_{2} A_{1}^{*}\right)\right] .
$$

Since $A_{1} A_{2}^{*}-A_{2} A_{1}^{*}$ is purely imaginary (it can also be zero when the incident wave is evanescent and no dissipation is present), $\operatorname{Re}\left[k\left(A_{1} A_{2}^{*}-A_{2} A_{1}^{*}\right)\right] \neq 0$, signifying that the superposition of the decaying and growing modes carry a finite power. We shall call this superposition as the interference of the evanescent waves. The total light transmittance of an opaque plasma slab occurs when the Poynting vector inside the slab is equal to that of the incident radiation, so that $\operatorname{Re}\left[k\left(A_{1} A_{2}^{*}-A_{2} A_{1}^{*}\right)\right]=S_{x 0}$. Figures (51) and (6) show the spatial distribution of the magnetic field modulus and $x$-component of time-averaged Poynting vector $S_{x}=\frac{1}{2} \operatorname{Re}\left[E_{y} B_{z}^{*}\right]$ for the case when surface wave is excited on a plasmaplasma interface. As one can see, the field amplitude grows dramatically in the first plasma layer (the magnitude of the magnetic field at the interface is more than fourteen times of that in the incident wave) to offset its exponential decay in the second to the level of the incident light. The $x$-component of the Poynting vector inside plasma regions is equal to that of the incident radiation-the energy of the field is perfectly transferred through the overcritical density plasma slab. For comparison, Fig. (17) shows the magnetic field amplitude for the case when there is no surface mode excited at the interface. Only exponentially 
decaying solution to the field equations is observed. It should be noted here that light can be transmitted through an opaque high-density slab even without surface waves present at the boundary, if the slab is thin enough. The effect of the evanescent wave interference is at play in this case too. The difference lies in the actual values of the expansion coefficients $A_{1}$ and $A_{2}$ in both cases. When there is no surface wave present, the coefficients are such that $\operatorname{Re}\left[k\left(A_{1} A_{2}^{*}-A_{2} A_{1}^{*}\right)\right]<S_{x 0}$, whereas if the surface wave is excited at the interface, the condition $\operatorname{Re}\left[k\left(A_{1} A_{2}^{*}-A_{2} A_{1}^{*}\right)\right]=S_{x 0}$ can be fulfilled and the absolute transparency of optically opaque plasma layer is observed.

\section{SURFACE WAVES ON A WARM PLASMA-PLASMA INTERFACE}

The results obtained in the previous sections are applicable to the cold plasma case, limiting its applications to mostly solid-state plasmas. In plasma states created through the interaction of the high power electromagnetic radiation with solid structures, the electron temperature can reach values in excess of $1 \mathrm{keV}$. Therefore, it is important to understand how the finite temperature modifies the dispersion relation of the surface wave and subsequently the anomalous light transmission through the dense plasma.

Kaw and McBride[12] have used hydrodynamic description to investigate the finite temperature dispersion relation for electromagnetic surface waves propagating on a plasmavacuum interface. We generalize their treatment for the case of surface waves on a warm plasma-plasma interface. The dispersion relation is found from solving the linearized set of warm fluid-Maxwell equations,

$$
\begin{array}{r}
\nabla \times \mathbf{E}=-\frac{1}{c} \frac{\partial \mathbf{B}}{\partial t} \\
\nabla \times \mathbf{B}=-\frac{4 \pi n_{0} e}{c} \tilde{\mathbf{v}}+\frac{1}{c} \frac{\partial \mathbf{E}}{\partial t} \\
\nabla \cdot \mathbf{B}=0 \\
\nabla \cdot \mathbf{E}=-4 \pi e \tilde{n} \\
\frac{\partial \tilde{n}}{\partial t}+\nabla \cdot n_{0} \tilde{\mathbf{v}}=0 \\
\frac{\partial \tilde{\mathbf{v}}}{\partial t}=-\frac{e}{m} \mathbf{E}-\frac{v_{T h}^{2}}{n_{0}} \nabla \tilde{n}
\end{array}
$$

where the subscript 0 indicates equilibrium quantities; $v_{T h}$ is electron thermal velocity; ions are assumed to be motionless. The above system of equations constitutes a closed set for 
the unknown electron density, hydrodynamic velocity and electromagnetic fields. Just like in the cold case, it can be readily solved assuming that density and field perturbations are in the form $\boldsymbol{\Psi}(x) \exp \left[i\left(k_{y} y-\omega t\right)\right]$, where $x$ is the coordinate into the plasma and $y$ runs along the interface. This gives the following expressions for the electromagnetic fields and electron density perturbation in both plasma regions assuming that the interface is at $x=0$ and omitting the explicit dependence on $\omega$ and $k_{y}$,

$$
\begin{array}{r}
\mathbf{E}_{(1)}=\mathbf{D}_{(1)} \exp \left[\alpha_{1} x\right]-\frac{4 \pi e A\left(1-\beta_{1}^{2}\right)}{\gamma_{1}^{2}-\alpha_{1}^{2}} \cdot\left(i k_{y} \hat{\mathbf{e}}_{\mathbf{y}}+\gamma_{1} \hat{\mathbf{e}}_{\mathbf{x}}\right) \exp \left[\gamma_{1} x\right], x<0 \\
\mathbf{E}_{(2)}=\mathbf{D}_{(2)} \exp \left[-\alpha_{2} x\right]-\frac{4 \pi e B\left(1-\beta_{2}^{2}\right)}{\gamma_{2}^{2}-\alpha_{2}^{2}} \cdot\left(i k_{y} \hat{\mathbf{e}}_{\mathbf{y}}-\gamma_{2} \hat{\mathbf{e}}_{\mathbf{x}}\right) \exp \left[-\gamma_{2} x\right], x>0 \\
\tilde{n}_{(1)}=A \exp \left[\gamma_{1} x\right], x<0 \\
\tilde{n}_{(2)}=B \exp \left[-\gamma_{2} x\right], x>0
\end{array}
$$

where $A, B, \mathbf{D}$ are unknown coefficients, $\beta=v_{T h} / c, \hat{\mathbf{e}}_{\mathbf{y}}, \hat{\mathbf{e}}_{\mathbf{x}}$ are the unit vectors in $y$ and $x$ directions respectively, $\gamma=\left(k_{y}^{2}+\frac{\omega_{p}^{2}-\omega^{2}}{v_{T h}^{2}}\right)^{1 / 2}, \alpha=\left(k_{y}^{2}+\frac{\omega_{p}^{2}-\omega^{2}}{c^{2}}\right)^{1 / 2} ;$ subscripts 1,2 designate both regions. As one can see the electromagnetic field in a bulk plasma is represented as a superposition of a transverse and longitudinal perturbations that are completely decoupled from each other. At this point we would like to bring reader's attention to the fact that just as in the cold plasma case, solution (7) describes decaying branches for the transverse fields as well as for a longitudinal field in the overcritical plasma region (described by the decay factor $\gamma_{2}$ in our notations). However, the density perturbation as well as the associated longitudinal electric field in the undercritical plasma region are described not by an evanescent/decaying branch, but rather by a propagating oscillatory function $\left(\gamma_{1}\right.$ takes on purely imaginary value in this region). The presence of non-decaying oscillations in the longitudinal field signifies the existence of a bulk plasma wave in the undercritical plasma region. The existence of the bulk plasmon stems from the linear wave conversion process that occurs at the plasma boundary [14]. In the conversion process, the surface plasma wave gives rise to a bulk longitudinal plasma mode that propagates away from the interface into the undercritical density plasma.

The dispersion relation for surface plasma waves on a warm plasma-plasma interface is found from the boundary conditions on the electromagnetic fields (tangential components of both fields e.q. $E_{y}$ and $B_{z}$ should be continuous across the interface). The two additional boundary conditions are obtained from the integration of the continuity equation and the 
equation of motion over $x$ coordinate in the limits $x= \pm \delta$ and setting subsequently $\delta=0$. Assuming that the density perturbation does not have any singularities at the interface (no surface charge present), the two additional boundary conditions have the following form,

$$
\begin{gathered}
\left.\frac{\tilde{n}}{n_{0}}\right|_{1}=\left.\frac{\tilde{n}}{n_{0}}\right|_{2} \\
\left.n_{0} \tilde{V}\right|_{1}=\left.n_{0} \tilde{V}\right|_{2}
\end{gathered}
$$

These are the continuity conditions for the electron density (normalized to its corresponding equilibrium value) and flux perturbations across the interface. In order to solve for all six unknowns in the system, the above four boundary conditions have to be also supplemented by the requirement that the divergence of the transverse part of the total electric field given by Eqs. (17) be equal to zero. Solving the system of six algebraic equations, one obtains

$$
\begin{gathered}
{\left[\left(\omega^{2}-\Delta\right) \sqrt{1-\omega^{2}+k_{y}^{2}}+\left(\omega^{2}-1\right) \sqrt{\Delta-\omega^{2}+k_{y}^{2}}\right]} \\
\times\left[\left(\omega^{2}-\Delta\right) \sqrt{\frac{1-\omega^{2}}{\beta^{2}}+k_{y}^{2}}+\Delta\left(\omega^{2}-1\right) \sqrt{\frac{\Delta-\omega^{2}}{\beta^{2}}+k_{y}^{2}}\right]=(\Delta-1)^{2} \omega^{2} k_{y}^{2},
\end{gathered}
$$

where $\Delta=\omega_{p 1}^{2} / \omega_{p 2}^{2}, \omega$ is normalized to the plasma frequency in the region 2 where $\epsilon<0$ and $k_{y}$ is normalized to the classical skin depth $\delta=c / \omega_{p 2}$. Eq. (91) is the general dispersion relation for surface plasma waves running on a warm homogeneous plasma-plasma interface. It is interesting to note that matching electromagnetic fields across the interface couples their longitudinal and transverse components, so that the resulting surface plasma mode is of mixed longitudinal-transverse type. Taking the limit $\Delta \rightarrow 0$ reduces this dispersion relation to that derived by Kaw [12] for the case of plasma-vacuum interface. The cold plasma dispersion relation is recovered from the limit $\beta \rightarrow 0$. Numerical solution of Eq. (9) shows that for $\beta_{1,2} \lesssim 0.02$, which corresponds to electron temperatures $204 \mathrm{eV}$ or lower, the cold plasma dispersion relation (31) yields solutions that are close to those given by the general expression (9), neglecting small imaginary parts in the eigenroots for a case of a warm plasma. The presence of imaginary values in the dispersion relation is directly linked to already mentioned conversion process. Because of the surface wave transformation into a bulk plasmon, its energy leaks out of the surface mode into a bulk plasma mode, which can be viewed as an effective dissipation of the surface plasmon, making the dispersion relation complex. It should be noted that the complex conjugate of the roots (with simultaneous reversal of the real parts of $\omega \rightarrow-\omega$ and $k_{y} \rightarrow-k_{y}$ ) are also solutions to the dispersion 
relation (9), which is physically related to the opposite process in which the bulk plasmon converts into the surface wave.

As plasma temperature gets higher, the eigenroots obtained from Eq. (9) can significantly deviate from those given in the cold plasma limit as can be seen from Fig. (8)). This fact should be taken into consideration when investigating a possible surface wave induced plasma heating channel in ICF experiments where the initial temperatures (due to slab interaction with laser prepulse) can exceed $1 \mathrm{keV}$ for ultra-intense laser pulses.

\section{TEMPERATURE EFFECTS AND THE ANOMALOUS LIGHT TRANSMIS- SION}

Let us consider a structure similar to that of the cold plasma case and characterized by the spatial density (dielectric constant) distribution shown in Fig. (11), but with non-zero electron temperature. A $p$-polarized plane electromagnetic wave is obliquely incident on this structure. In both plasma regions, the electromagnetic fields $\mathbf{B}=\left(0,0, B_{z}\right), \mathbf{E}=\left(E_{x}, E_{y}, 0\right)$ are the solution to the following system of equations:

$$
\begin{array}{r}
\beta^{2} \frac{d^{2} E_{x}}{d x^{2}}+\left(\frac{\omega^{2}}{c^{2}} \epsilon-k_{y}^{2}\right) E_{x}=i k_{y}\left(1-\beta^{2}\right) \frac{d E_{y}}{d x} \\
\frac{d^{2} E_{y}}{d x^{2}}+\left(\frac{\omega^{2}}{c^{2}} \epsilon-\beta^{2} k_{y}^{2}\right) E_{y}=i k_{y}\left(1-\beta^{2}\right) \frac{d E_{x}}{d x} \\
\epsilon \frac{d}{d x}\left(\frac{1}{\epsilon} \frac{d B_{z}}{d x}\right)+\frac{\omega^{2}}{c^{2}}\left(\epsilon-\sin ^{2} \theta\right) B_{z}=0
\end{array}
$$

where $k_{y}=\omega / c \sin \theta$ and $\beta^{2}=3 v_{T h}^{2} / c^{2}$ (the factor of 3 originates from the adiabatic equation of state) and $\epsilon=1-\omega_{p}^{2} / \omega^{2}$. The system of equations (10) can be readily obtained from the initial set of Maxwell and warm fluid equations. It should be emphasized that Eqs. (10) are only valid for not too high electron temperatures, so that the electron mean free path is smaller than the spatial inhomogeneity of the field inside the plasma. Otherwise, the hydrodynamic treatment is not adequate and one has to invoke kinetic description, as in a problem of anomalous skin effect [5, 11]. A general solution to the above system is

$$
\begin{array}{r}
B_{z}=A_{1} e^{i k x}+A_{2} e^{-i k x} \\
E_{x}=-\frac{k_{y}}{k_{0} \epsilon}\left(A_{1} e^{i k x}+A_{2} e^{-i k x}\right)-\frac{k_{0}}{k_{y}}\left(C_{1} e^{i k_{p} x}+C_{2} e^{-i k_{p} x}\right) \\
E_{y}=\frac{k}{k_{0} \epsilon}\left(A_{1} e^{i k x}-A_{2} e^{-i k x}\right)-\frac{k_{0}}{k_{p}}\left(C_{1} e^{i k_{p} x}-C_{2} e^{-i k_{p} x}\right),
\end{array}
$$


where $k_{0}=\omega / c, k=k_{0} \sqrt{\left(\epsilon-\sin ^{2} \theta\right)}, k_{p}=k_{0} \sqrt{\left(\epsilon-\beta^{2} \sin ^{2} \theta\right)} / \beta$. Coefficients $A_{1,2}$ represent transverse part of the total field and $C_{1,2}$ represent its longitudinal/electrostatic part. The electromagnetic fields in the vacuum regions $(x<-d$ and $x>a)$ have the following form,

$$
\begin{array}{r}
B_{z}=E_{0} e^{i k_{0} \cos \theta x}+R e^{-i k_{0} \cos \theta x}, x<-d \\
E_{x}=-E_{0} \sin \theta e^{i k_{0} \cos \theta x}-R \sin \theta e^{-i k_{0} \cos \theta x}, x<-d \\
E_{y}=E_{0} \cos \theta e^{i k_{0} \cos \theta x}-R \cos \theta e^{-i k_{0} \cos \theta x}, x<-d \\
B_{z}=T e^{i k_{0} \cos \theta x}, \quad x>a \\
E_{x}=-T \sin \theta e^{i k_{0} \cos \theta x}, \quad x>a \\
E_{y}=T \cos \theta e^{i k_{0} \cos \theta x}, \quad x>a
\end{array}
$$

where $T$ and $R$ designate the field components for the transmitted and reflected waves. By matching the solutions at all boundaries (requiring continuity of $E_{y}, B_{z}, n_{0} \tilde{V}_{x}$ and $\tilde{n} / n_{0}$ across interfaces), one obtains a system of ten equations (not shown here) for the unknown coefficients. It should be noted that in Ref. [7] the authors used similar approach to investigate the anomalous transmission of light through a structure consisting of an overdense warm plasma slab spaced between two underdense plasma layers. However, the choice of boundary conditions on the fields used by the authors, specifically the continuity of the spatial derivative of the $x$-component ( $z$-component in their notations) of the electric field along $x$ leads to non-physical solutions in which the transmission $T=\left|\frac{T}{E_{0}}\right|^{2}$ or reflection $R=\left|\frac{R}{E_{0}}\right|^{2}$ coefficients can be greater than one. Boundary conditions employed in current work lead to solutions that always satisfy the identity $|R|^{2}+|T|^{2}=1$ (which is the conservation of energy for a plain-wave case for a non-dissipative system). The resulting expressions for the transmission/reflection coefficients are extremely bulky functions of electron temperature, electromagnetic wave incidence angle, its frequency, both plasma layers electron densities and thicknesses.

As mentioned in the previous section, the dispersion relation for the surface plasma waves at non-zero temperatures can significantly deviate from that obtained in the cold plasma limit. Since the anomalous transmission of light is due to the excitation of the surface plasmons, the resulting transmission coefficient exhibits thermal effects that are not seen in cold 
plasmas. Figure (97) shows the dependence of the transmission coefficient on the thermal electron velocity $v_{T h}=\sqrt{T / m_{e}}$. As one can see, it shows high frequency oscillations in the electron temperature space. The presence of these regular oscillations in the transmission of the system is related to the linear wave conversion processes that occur at the plasma boundaries. The surface wave excites a bulk longitudinal plasma mode propagating in the underdense plasma layer away from the plasma-plasma boundary. When it reaches the vacuum-plasma interface, the longitudinal plasma wave converts into a transverse propagating electromagnetic wave, which interferes with the reflected light. Thus, in the general case the interference between the incident/reflected fields, surface plasma wave induced fields and the fields created through the linear conversion processes determine the transmission.

The temperature oscillations can be understood using the following simplified model. The surface plasma wave excites a bulk plasmon propagating at angle $\theta^{\prime}$ to the surface normal. This angle can be found from matching the $y$ components of both modes' wave numbers to give:

$$
\sin \theta^{\prime}=\frac{\beta}{\sqrt{\epsilon}} \sin \theta
$$

The plasma wave propagates the distance $L=d / \cos \theta^{\prime}$ with phase velocity $v_{p h}=c \beta / \sqrt{\epsilon}$. Therefore, the phase difference $\Delta \phi=\omega \Delta t$ between the reflected light and that emitted through the conversion process is,

$$
\Delta \phi=\frac{k_{0} d \sqrt{\frac{\epsilon}{\beta^{2}}}}{\sqrt{1-\frac{\beta^{2}}{\epsilon} \sin ^{2} \theta}} .
$$

When the phase difference is equal to a multiple of $2 \pi$, both components interfere constructively giving rise to large reflection and thus low transmission. Therefore, one arrives at a following temperature condition at which minimal light transmission occurs through the system,

$$
\beta=\sqrt{\frac{1}{2} \epsilon \csc ^{2} \theta-\frac{\epsilon \csc ^{2} \theta \sqrt{\pi^{2} n^{2}-d^{2} k_{0}^{2} \sin ^{2} \theta}}{2 \pi n}}, n=1,2,3, \ldots
$$

With a simple estimate given by Eq. (14), we are able to recover some of the minima in the transmission coefficient obtained from the complete solution of Eqs. (9). Other minima in the transmission coefficient, which are not described by Eq. (14) are due to other conversion 
processes like the incident wave transformation into a longitudinal plasma mode at the vacuum-plasma interface that travels to the plasma-plasma interface, gets reflected (exciting a surface mode in a process) and travels back to the plasma-vacuum interface converting to the transverse electromagnetic wave. All these conversion processes contribute to the transmission properties of the double-plasma system, making it highly oscillatory function of not only the electron temperature but also the underdense plasma layer thickness $d$ as can be seem from Fig. (10). The multiple peaks present in the transmission coefficient in this case can also be explained through the constructive interference between the reflected electromagnetic field and the field created as a result of the linear wave conversion process. Since the phase difference $\Delta \phi$ also depends on the underdense layer thickness, one can arrive at a similar condition for the plasma thickness $d$ at which minimal light transmission occurs through the system,

$$
d=\frac{2 \pi n \sqrt{1-\frac{\beta^{2} \sin ^{2} \theta}{\epsilon}}}{k_{0} \sqrt{\frac{\epsilon}{\beta^{2}}}}, n=1,2,3, \ldots
$$

A simple estimate given by Eq. (15) can also recover the minima in the transmission coefficient as a function of the undercritical density plasma layer thickness for a case of non-zero electron temperature. As the plasma temperature increases, the resonant condition for the excitation of the surface wave changes according to the dispersion relation given by Eq. (9), shifting the peak in the transmission coefficient to smaller angles. A distinct difference between the zero temperature and a warm plasma case lies in a fact that there is only a single peak in the transmission coefficient (as a function of the undercritical density plasma layer thickness) in the cold plasma case, whereas the number of transmission peaks in a warm plasma is quite large. Unlike the cold plasma case where the energy of the external radiation is transferred through the constructive interference of evanescent fields, in a warm plasma case the superposition of the propagating longitudinal plasma wave fields (in a undercritical density plasma slab) and the evanescent transverse electromagnetic fields determine the amount of the transmitted light as can be seen from figure (11), where the $x$ components of time-averaged total (which includes the contribution of plasma electrons) Poynting vector and the real part of the electric field are shown. As expected, the electromagnetic field has a form of the superposition of the evanescent and propagating/oscillatory branches and under resonant condition the energy is totally transferred through the system. In addition, the 
results of our calculations show that in a warm underdense plasma layer the energy is mainly carried by the longitudinal electron plasma oscillations, facilitating the total transparency of the considered dual-layer system for quite large thicknesses of an undercritical density plasma slab.

\section{CONCLUSIONS}

It is shown here that overcritical density plasma can be made transparent to external radiation. The total transparency can be achieved even with a single surface mode excited on a plasma-plasma boundary. The anomalous transmission is explained through the interference effect between the evanescent fields of the incident electromagnetic radiation and those associated with the surface plasmons. Superposition of two (decaying and growing) evanescent modes can carry the power of the incident radiation over to the other side of a high-density plasma slab, making it totally transparent to light in a case when irreversible dissipation is absent. We have also shown that the excitation of the longitudinal plasma waves creates a multiplicity of additional resonances corresponding to the absolute transparency. We suggest that the mechanism investigated in this paper may be responsible for the anomalous transparency of metals observed recently in a number of experiments [8, 17].

The role of dissipation effects is twofold, depending on possible applications of the surface wave phenomenon. In ICF experiments where high absorption of the incident radiation is sought, the presence of irreversible dissipation is necessary for effective heating of a fuel pellet through damping of the surface plasma waves. If dissipation is absent, the energy of the incident light will be totally re-emitted by these waves back into vacuum. If on the other hand a high transmission of light is needed, the dissipation plays a detrimental role, limiting the transmitting ability of the system. Therefore, a close control over a number of physical parameters (electron density, temperature, thickness) of the double-layer system is needed for successful technological implementation of the surface wave effect.

The final point we would like to mention is related to the fact that since the Maxwell equations are invariant under reversal of time and space coordinates, the order at which the incident electromagnetic wave encounters both plasma layers (an undercritical density layer followed by that with overcritical density or vice versa) does not effect its transmission ability. 


\section{Acknowledgments}

This work is in part supported by NIH(CA78331), Strawbridge Family Foundation, Varian medical systems and NSERC Canada.

[1] Yu. M. Aliev, O M. Gradov, and A. Yu. Kyrie. Total absorption of electromagnetic radiation in the dense inhomogeneous plasma. Phys. Review A, 15:2120-2122, 1977.

[2] Y. Bliokh, J. Felsteiner, and Y. Slutsker. Total absorption of electromagnetic wave by an overdense plasma. Phys. Rev. Lett., 95:165003-1-165003-4, 2005.

[3] J. M. Dawson and J. Oberman. High frequency conductivity and the emission and absorption coefficients of a fully ionized plasma. Phys. Fluids, 5:517, 1962.

[4] N. G. Denisov. On a singularity of the field of an electromagnetic wave propagated in an inhomogeneous plasma. Sov. Phys.-JETP, 4:544, 1957.

[5] R. Dragila and E. G. Gamaliy. Surface waves in the regime of anomalous skin effect. Phys. Rev. A, 15:6828-6833, 1991.

[6] R. Dragila, B. Luther-Davies, and S. Vukovic. High transparency of classically opaque mettalic films. Phys. Rev. Lett., 55:1117-1120, 1985.

[7] R. Dragila and S. Vukovic. Surface-wave-induced high transparency of an overdense warm plasma layer. Optics Letters, 12:573-575, 1987.

[8] N. Fang, Hyesog Lee, Cheng Sun, and Xiang Zhang. Sub-diffraction-limited optical imaging with a silver superlens. Science, 308:534, 2005.

[9] D. Forslund, J. Kindel, K. Lee, E. Lindman, and R. Morse. Theory and simulation of resonant absorption in a hot plasma. Phys. Rev. A, 11:679, 1975.

[10] J. P. Freidberg, R. W. Mitchell, R. L. Morse, and L. I. Rudsinski. Resonant absorption of laser light by plasma targtes. Phys. Rev. Lett., 28:795-799, 1972.

[11] S. Ichimaru. Basic principles of plasma physics: a statistical approach. W. A. Benjamin, Reading, MA, 1973.

[12] P. K. Kaw and J. B. McBride. Surface waves on a plasma half space. Phys. Fluids, 13:17841790, 1970.

[13] J. M. Kindel, K. Lee, and E. L. Lindman. Surface wave absorption. Phys. Rev. Lett., 34:134- 
$138,1975$.

[14] A. N. Kondratenko. Penetration of electromagnetic fields into plasma. Atomizdat, In Russian, 1979.

[15] L. D. Landau and E. M. Lifshits. Quantum Mechanics: non-relativistic theory. Pergamon Press, Oxford, 1977.

[16] A. B. Langdon. Nonlinear inverse bremsstrahlung and heated electron distributions. Phys. Rev. Lett., 44:575, 1980.

[17] J. B. Pendry. Negative refraction makes a perfect lens. Phys. Rev. Lett., 85:3966-3969, 2000.

[18] V. A. Podolskiy, N. A. Kuhta, and G. W. Milton. Optimizing the superlense: Manipulating geometry to enhance the resolution. Appl. Phys. Lett., 87:231113, 2005.

[19] A. W. Trivelpiece and R. W. Gould. Space charge waves in cylindrical plasma columns. J. Appl. Phys., 30:1784-1793, 1959.

[20] V. G. Veselago. The electrodynamics of substances with simultaneously negative values of eps and mu. Sov. Phys. Usp., 10:509, 1968.

[21] S. Vukovic and R. Dragila. Total transparency of an overdense plasma layer. J. Opt. Soc. Am. B, 3:1585-1587, 1986.

\section{Figures}




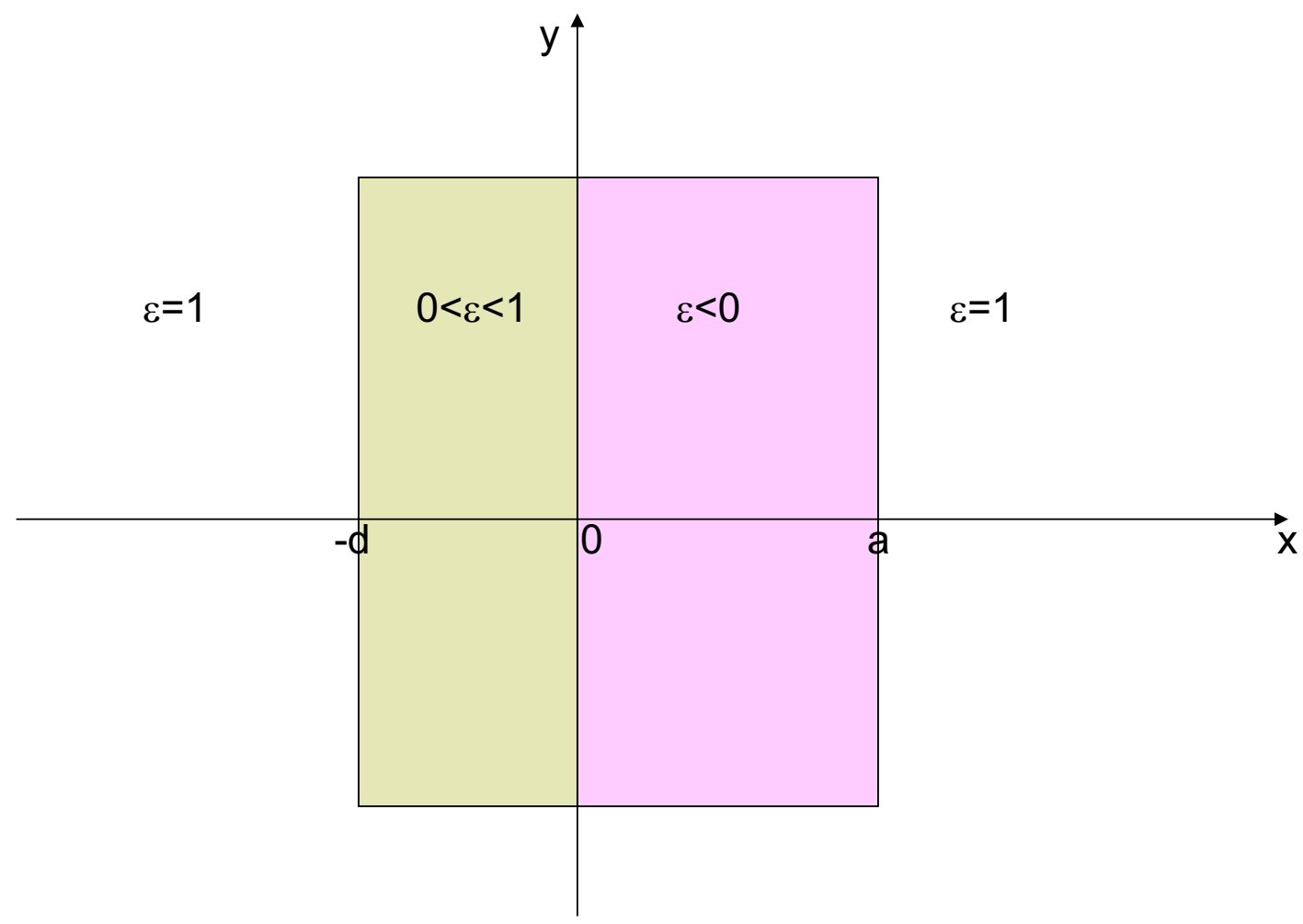

FIG. 1: Schematic diagram of the spatial density (dielectric constant) distribution.

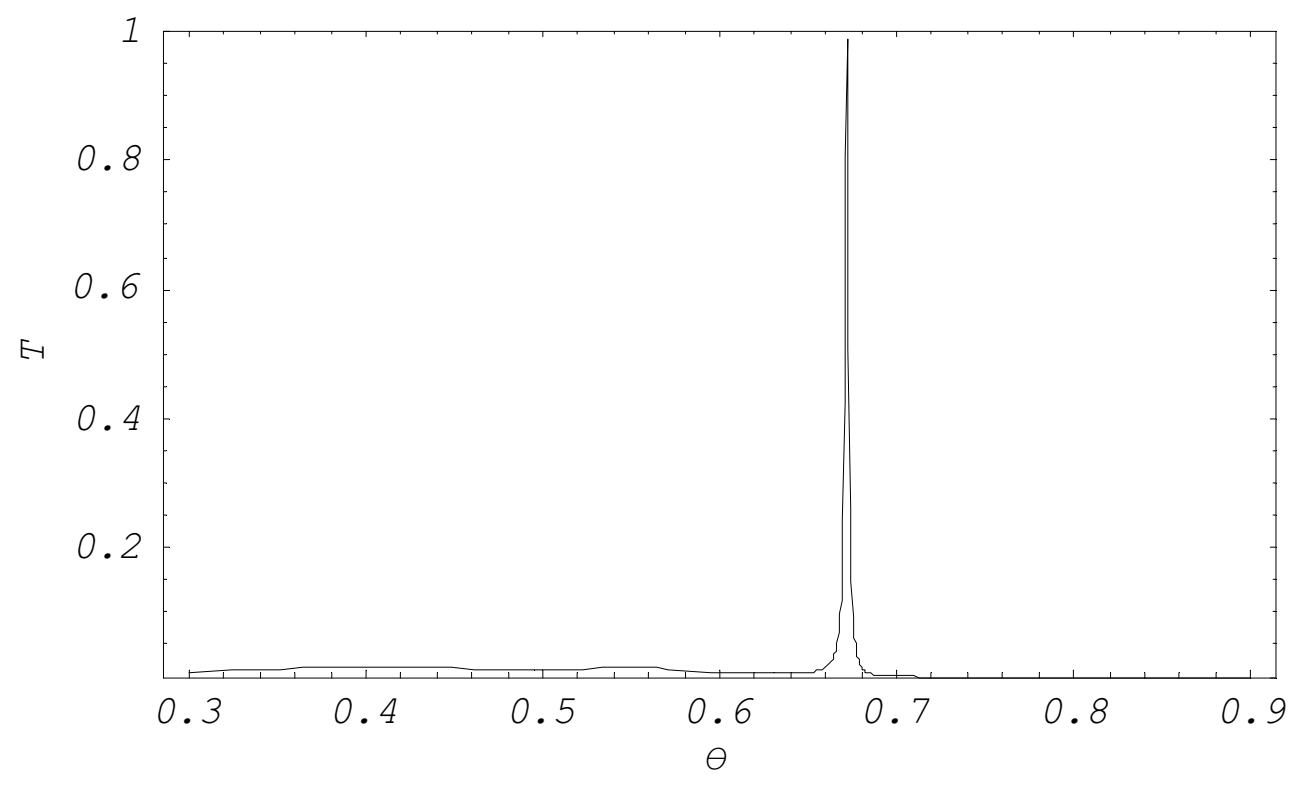


FIG. 2: Transmission coefficient as a function of incidence angle. A double-layer plasma system becomes completely transparent at the incidence angle $\theta=0.671955$. Undercritical and overcritical plasma slabs dielectric constants and thicknesses are $\epsilon_{1}=0.3428, d=27 * c / \omega_{p 2}$ and $\epsilon_{2}=-2.97$, $a=3.12 * c / \omega_{p 2}$ correspondingly. External radiation frequency $\omega / \omega_{p 2}=0.5019$.

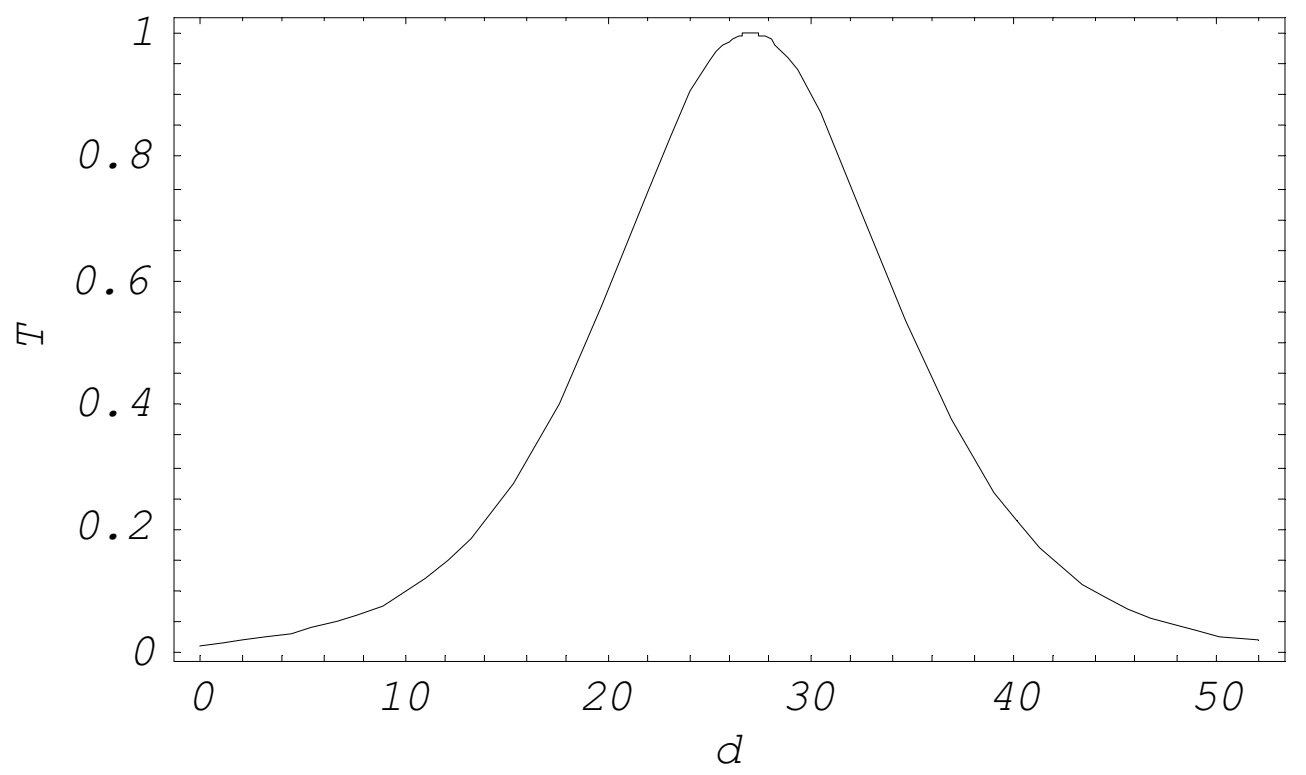

FIG. 3: Transmission coefficient as a function of a underdense plasma layer thickness. Overcritical plasma slab dielectric constant and thickness are $\epsilon_{2}=-2.97, a=3.12 * c / \omega_{p 2}$ correspondingly. Underdense plasma layer dielectric constant is $\epsilon_{1}=0.3428$. External radiation frequency $\omega / \omega_{p 2}=0.5019$. Incidence angle $\theta=0.671955$. 


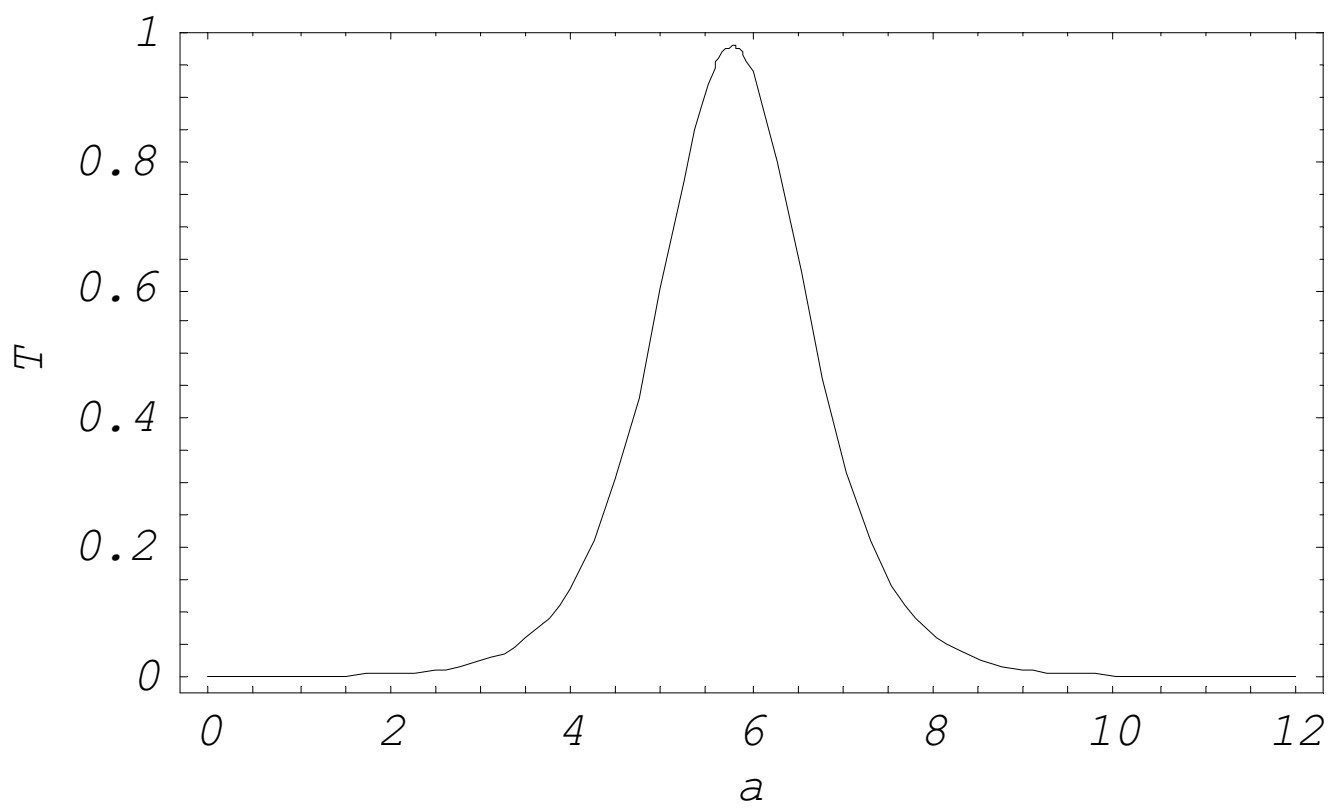

FIG. 4: Transmission coefficient as a function of an overdense plasma layer thickness. Overcritical plasma slab dielectric constant is $\epsilon_{2}=-2.97$. Underdense plasma layer dielectric constant and thickness are $\epsilon_{1}=0.3428$ and $d=50 * c / \omega_{p 2}$ correspondingly. External radiation frequency $\omega / \omega_{p 2}=0.5019$. Incidence angle $\theta=0.671955$.

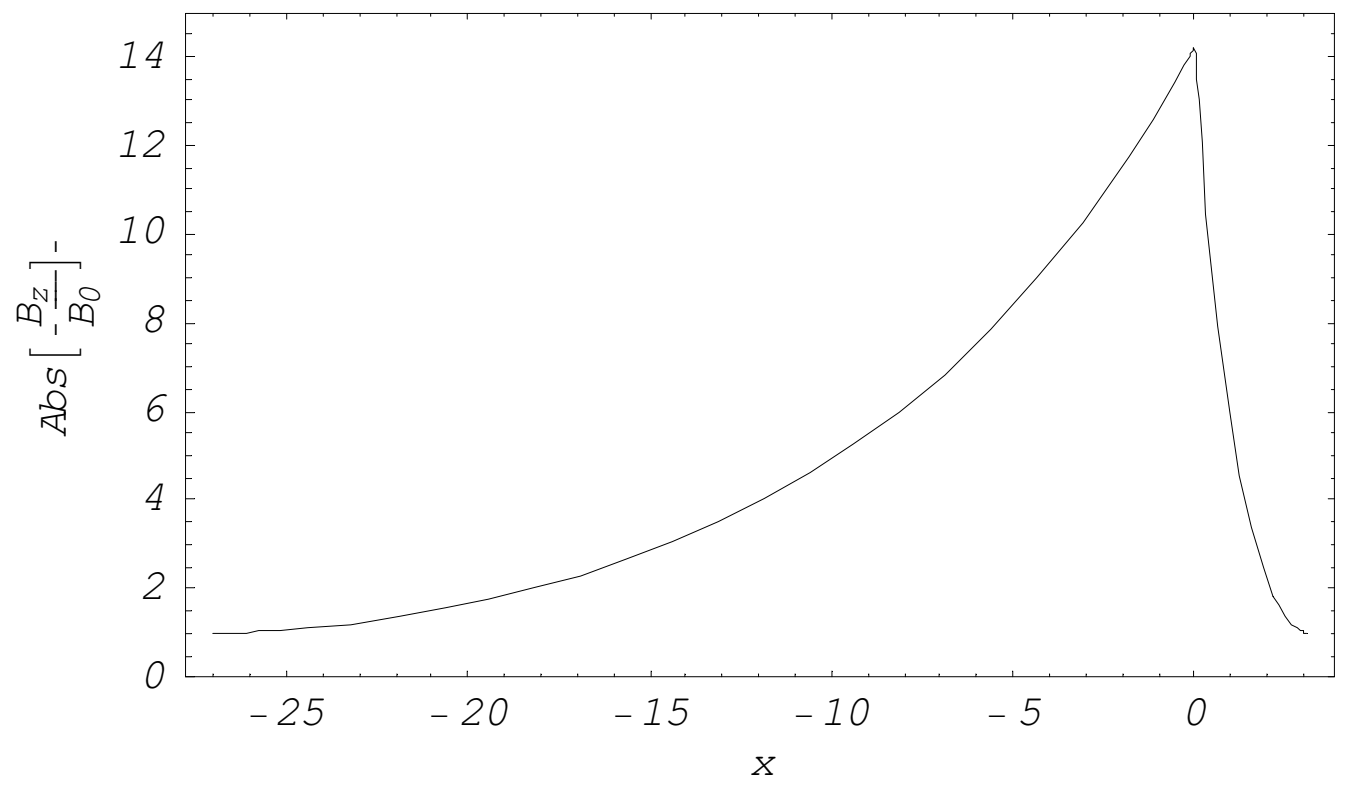


FIG. 5: Spatial distribution of an absolute value of the magnetic field associated with a single surface wave present on a plasma-plasma boundary. Undercritical and overcritical plasma slabs dielectric constants and thicknesses are $\epsilon_{1}=0.3428, d=27 * c / \omega_{p 2}$ and $\epsilon_{2}=-2.97, a=3.12 * c / \omega_{p 2}$ correspondingly. External radiation frequency and angle of incidence are $\omega / \omega_{p 2}=0.5019, \theta=$ 0.671955 correspondingly. $B_{0}$ is the magnitude of the magnetic field in the incident wave.

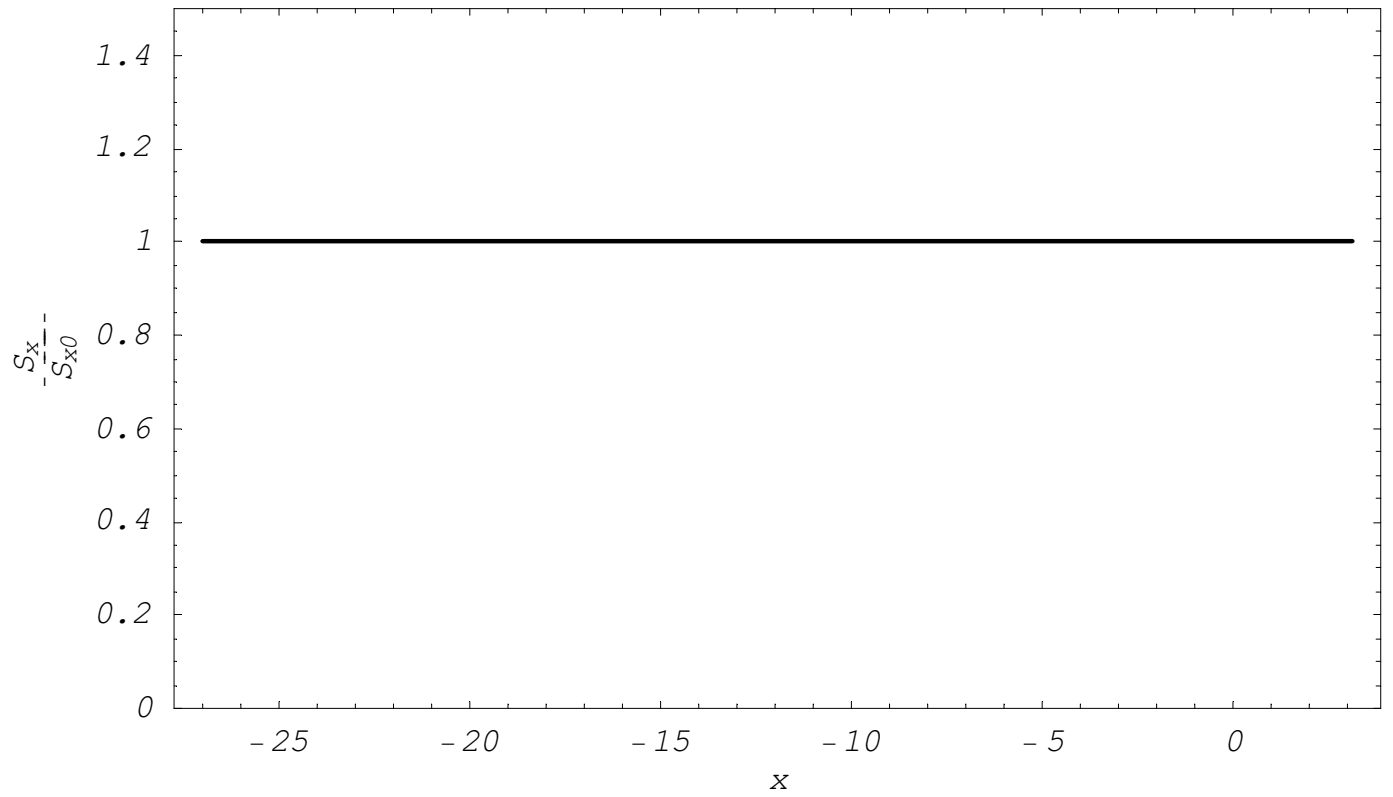

FIG. 6: Spatial distribution of the $x$-component of the Poynting vector $S_{x}$, normalized to the $x$-component of the Poynting vector of the incident wave $S_{x 0}=1 / 2 E_{0}^{2} \cos \theta$. 


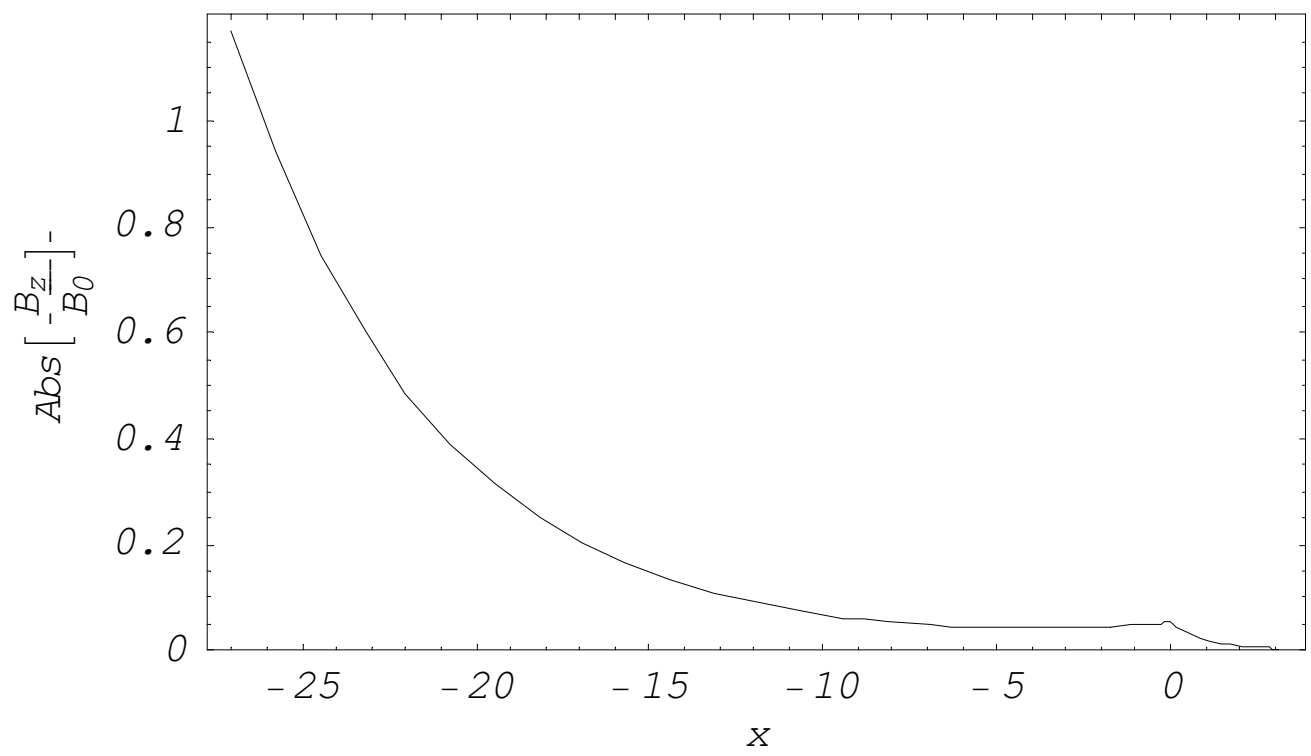

FIG. 7: Spatial distribution of an absolute value of the magnetic field when there is no surface wave present on a plasma-plasma boundary. Undercritical and overcritical plasma slabs dielectric constants and thicknesses are $\epsilon_{1}=0.3428, d=27 * c / \omega_{p 2}$ and $\epsilon_{2}=-2.97, a=3.12 * c / \omega_{p 2}$ correspondingly. External radiation frequency and angle of incidence are $\omega / \omega_{p 2}=0.5019, \theta=0.75$ correspondingly. $B_{0}$ is the magnitude of the magnetic field in the incident wave.

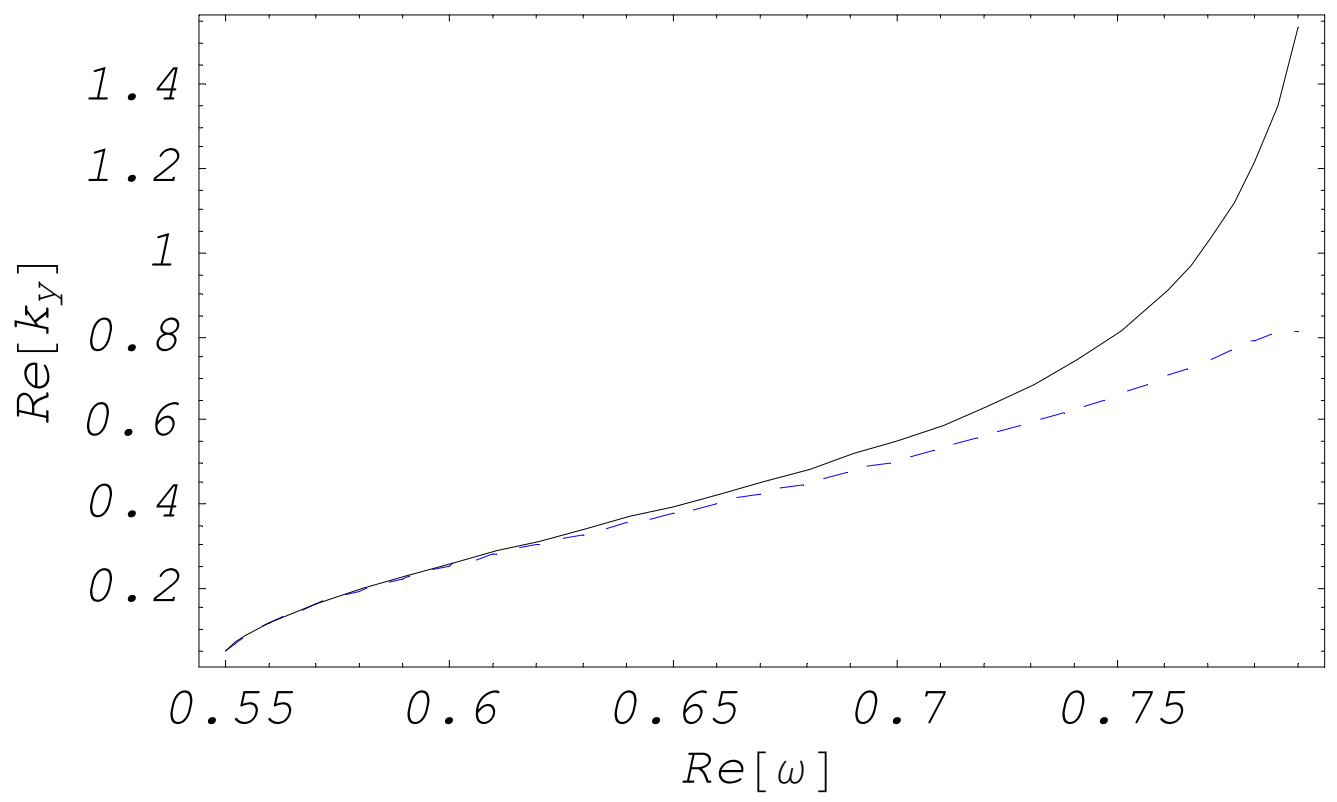


FIG. 8: Dispersion relation for surface waves on plasma-plasma interface. The dashed line is the numerical solution of Eq. (3) for $\Delta=0.3, \beta_{1}=\beta_{2}=0.1$. The solid line corresponds to the cold plasma limit with $\Delta=0.3$. $\omega$ is normalized to the plasma frequency of an overcritical density region and $k_{y}$ is normalized to its classical skin depth $c / \omega_{p 2}$

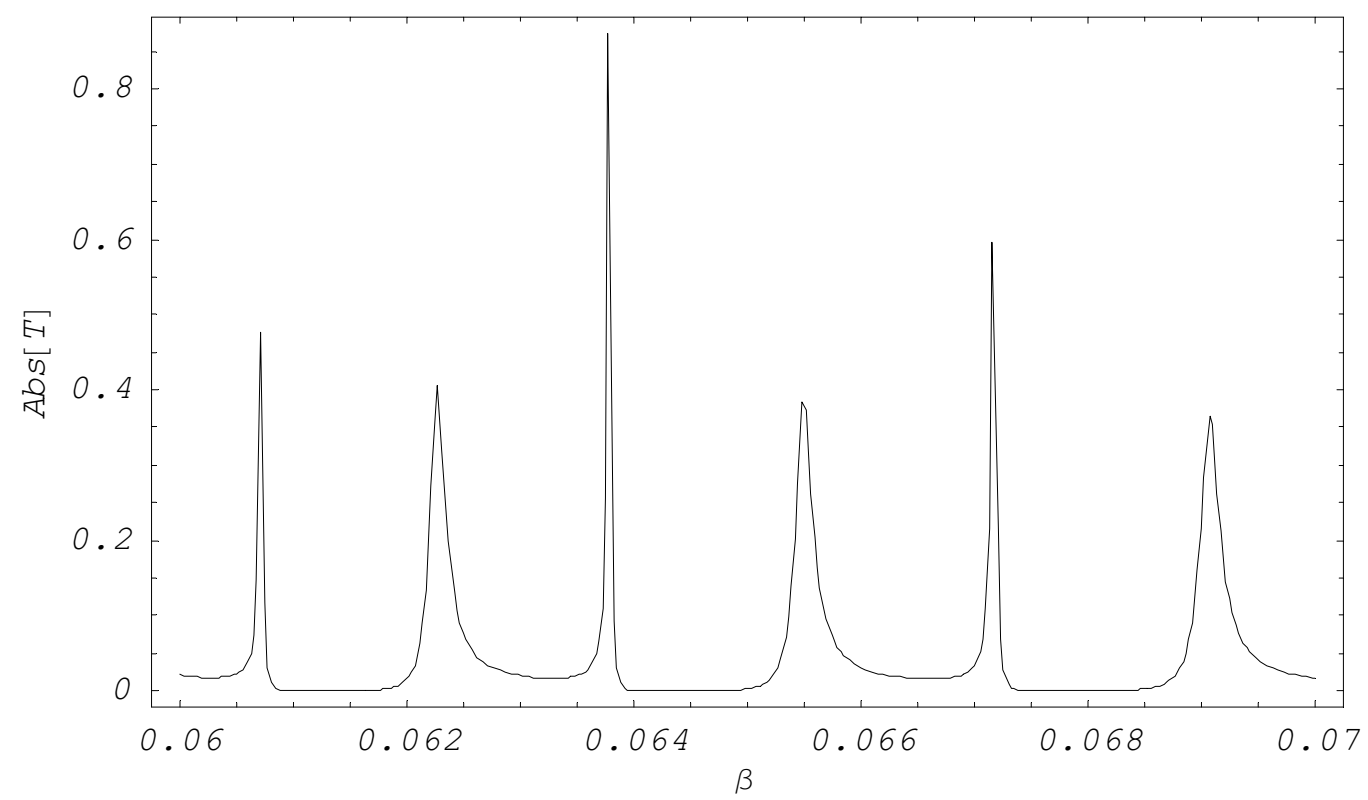

FIG. 9: Transmission coefficient as a function of electron temperature $\beta=\sqrt{3 T / m_{e} c^{2}}$. Undercritical and overcritical plasma slabs dielectric constants and thicknesses are $\epsilon_{1}=0.3428, d=27 * c / \omega_{p 2}$ and $\epsilon_{2}=-2.97, a=3.12 * c / \omega_{p 2}$ correspondingly. External radiation frequency and angle of incidence are $\omega / \omega_{p 2}=0.5019, \theta=0.671955$ correspondingly. 


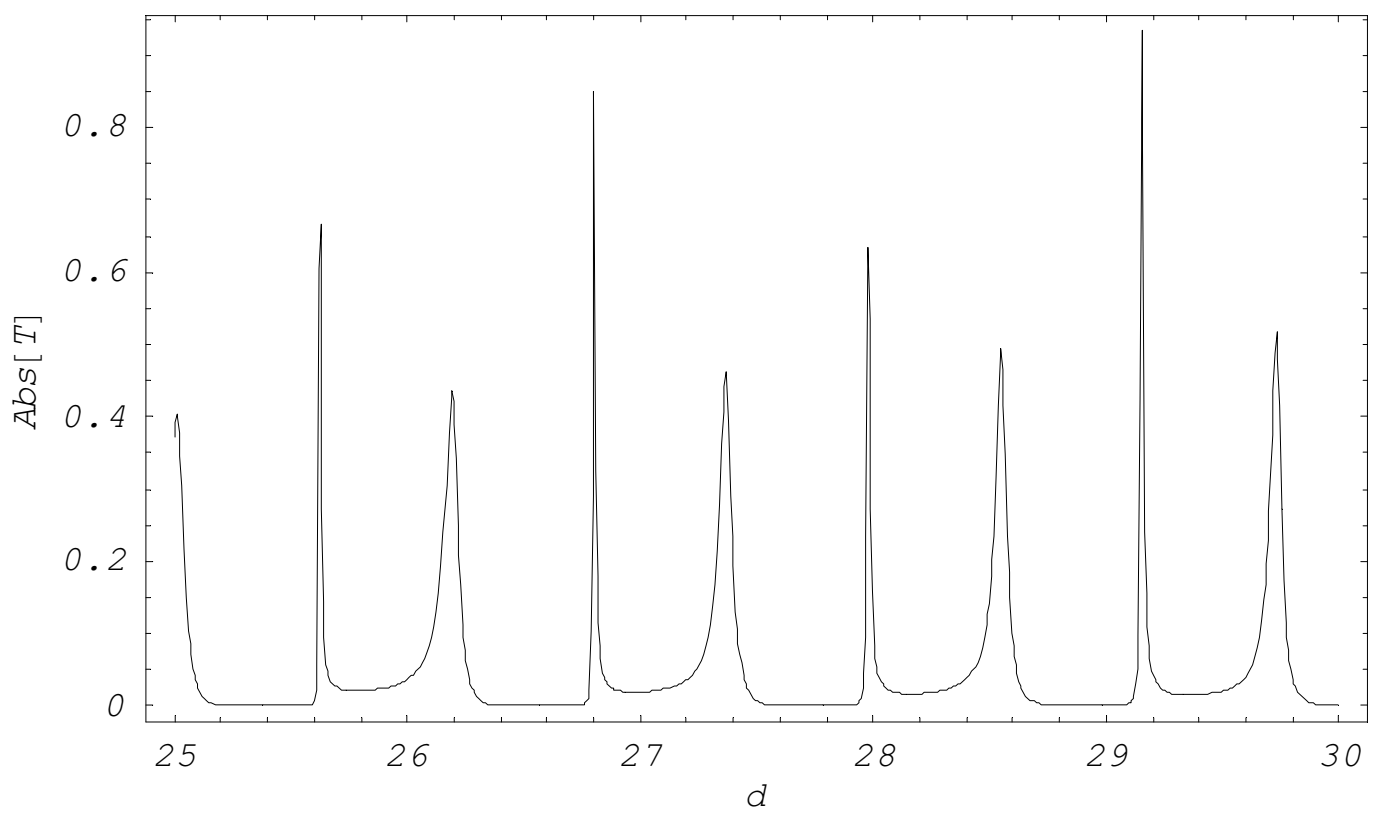

FIG. 10: Transmission coefficient as a function of a underdense plasma layer thickness $d$ for plasma temperature parameter $\beta=0.055$. Overcritical plasma slab dielectric constant and thickness are $\epsilon_{2}=-2.97, a=3.12 * c / \omega_{p 2}$ correspondingly. Underdense plasma layer dielectric constant is $\epsilon_{1}=0.3428$. External radiation frequency $\omega / \omega_{p 2}=0.5019$. Incidence angle $\theta=0.671955$.

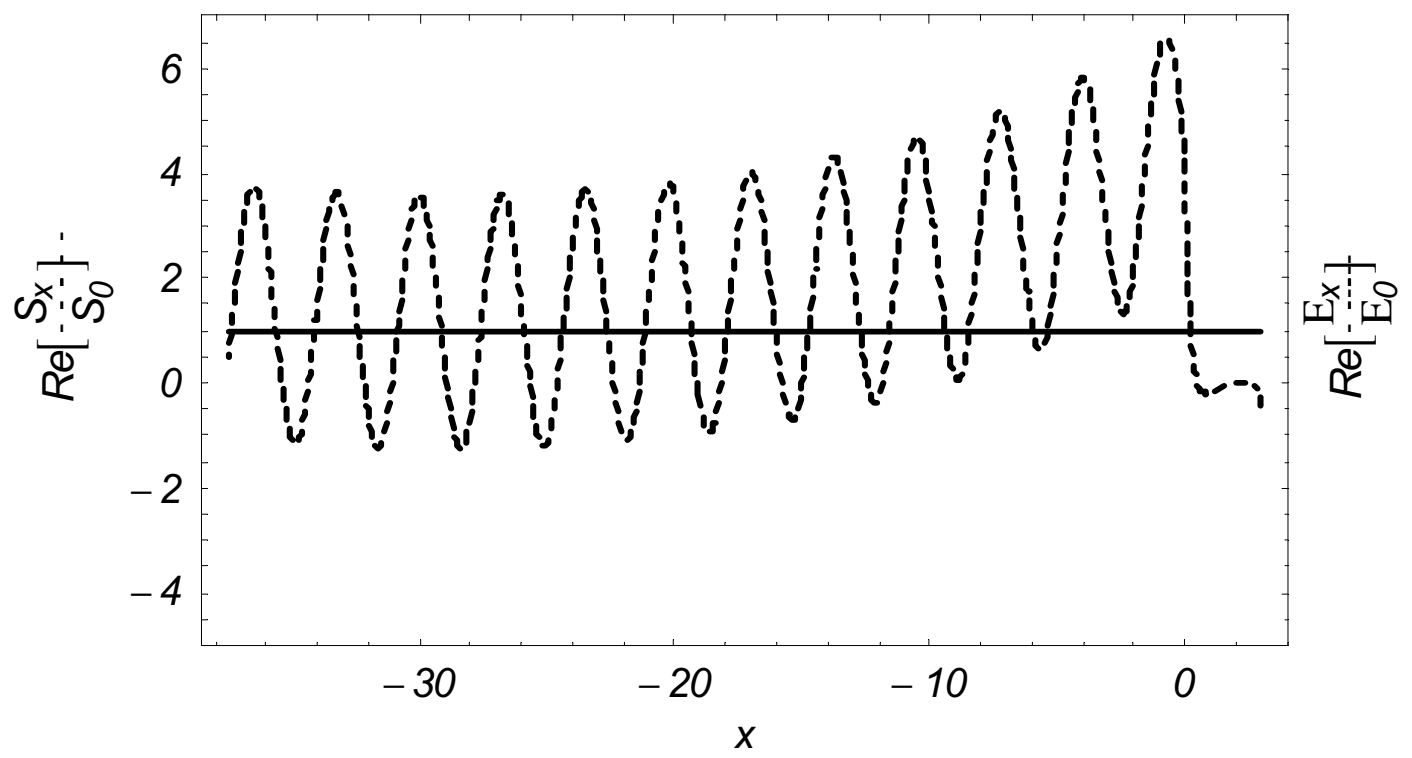


FIG. 11: Spatial distributions of the $x$-components of the Poynting vector $S_{x}$ (solid line) and the total electric field (dashed line) inside plasma layers for plasma temperature parameter $\beta=$ 0.15. Overcritical plasma slab dielectric constant and thickness are $\epsilon_{2}=-2.97, a=3 . * c / \omega_{p 2}$ correspondingly. Underdense plasma layer dielectric constant and thickness are $\epsilon_{1}=0.3428, d=$ $37.5 * c / \omega_{p 2}$. External radiation frequency $\omega / \omega_{p 2}=0.5019$. Incidence angle $\theta=0.64516$. 